\title{
Mapping the neutral atomic hydrogen gas outflow in the restarted radio galaxy $3 \mathrm{C} 236^{\star}$
}

\author{
R. Schulz ${ }^{1}$, R. Morganti ${ }^{1,2}$, K. Nyland ${ }^{3}$, Z. Paragi ${ }^{4}$, E. K. Mahony ${ }^{5,6}$, and T. Oosterloo ${ }^{1,2}$ \\ 1 ASTRON, Netherlands Institute for Radio Astronomy, Postbus 2, 7990 AA Dwingeloo, The Netherlands \\ e-mail: schulz@astron.nl \\ 2 Kapteyn Astronomical Institute, University of Groningen, PO Box 800, 9700 AV Groningen, The Netherlands \\ 3 National Radio Astronomy Observatory, Charlottesville, VA 22903, USA \\ 4 Joint Institute for VLBI ERIC, Postbus 2, 7990 AA Dwingeloo, The Netherlands \\ 5 Sydney Institute for Astronomy, School of Physics A28, The University of Sydney, NSW 2006, Australia \\ 6 ARC Centre of Excellence for All-Sky Astrophysics (CAASTRO), Australia
}

Received 26 March 2018 / Accepted 14 June 2018

\begin{abstract}
The energetic feedback that is generated by radio jets in active galactic nuclei (AGNs) has been suggested to be able to produce fast outflows of atomic hydrogen (HI) gas, which can be studied in absorption at high spatial resolution. We have used the Very Large Array (VLA) and a global very long baseline interferometry (VLBI) array to locate and study in detail the H I outflow discovered with the Westerbork Synthesis Radio Telescope (WSRT) in the restarted radio galaxy 3C 236. Based on the VLA data, we confirm a blueshifted wing of the HI with a width of $\sim 1000 \mathrm{~km} \mathrm{~s}^{-1}$. This H I outflow is partially recovered by the VLBI observation. In particular, we detect four clouds with masses of $0.28-1.5 \times 10^{4} M_{\odot}$ with VLBI that do not follow the regular rotation of most of the HI. Three of these clouds are located, in projection, against the nuclear region on scales of $\lesssim 40 \mathrm{pc}$, while the fourth is cospatial to the southeast lobe at a projected distance of $\sim 270 \mathrm{pc}$. Their velocities are between 150 and $640 \mathrm{~km} \mathrm{~s}^{-1}$ blueshifted with respect to the velocity of the disk-related HI. These findings suggest that the outflow is at least partly formed by clouds, as predicted by some numerical simulations, and that it originates already in the inner (few tens of pc) region of the radio galaxy. Our results indicate that the entire outflow might consist of many clouds, possibly with comparable properties as those clearly detected, but distributed at larger radii from the nucleus where the lower brightness of the lobe does not allow us to detect them. However, we cannot rule out a diffuse component of the outflow. Because 3C 236 is a low-excitation radio galaxy, it is less likely that the optical AGN is able to produce strong radiative winds. This leaves the radio jet as the main driver for the H I outflow.
\end{abstract}

Key words. galaxies: active - galaxies: jets - galaxies: individual: 3C 236 - galaxies: ISM - ISM: jets and outflows techniques: high angular resolution

\section{Introduction}

The evolution of galaxies is considered to be strongly linked to that of their central supermassive black holes (SMBH). The required feedback is commonly explained by a phase of enhanced activity related to the SMBH (e.g., Heckman \& Best 2014; Kormendy \& Ho 2013). An active galactic nucleus (AGN) can affect the interstellar medium (ISM) by heating up and expelling gas, which hinders star formation and the accretion of matter onto the SMBH (e.g., Silk \& Rees 1998; Di Matteo et al. 2005; Croton et al. 2006; McNamara \& Nulsen 2007). Prominent observational signatures include outflows of ionized, molecular, and atomic gas. These signatures have been associated with a number of AGN at a range of redshifts. The highest outflow rates have been determined for the cold ISM gas (molecular and atomic). The different possible drivers of these outflows include the radio jets launched in some AGN. The complex interplay between the AGN and the ISM requires detailed observational and theoretical studies of each phase of the outflowing gas (see reviews by Veilleux et al. 2005; Fabian 2012; Alexander \& Hickox 2012; Wagner et al. 2016; Tadhunter 2016;

\footnotetext{
* The VLBI image and cube are only available at the CDS via anonymous ftp to cdsarc.u-strasbg.fr (130.79.128.5) or via https://cdsarc.u-strasbg.fr/viz-bin/qcat?]/A+A/617/A38
}

Harrison 2017; Morganti 2017 and references therein). Here, we focus on the outflows of neutral atomic hydrogen ( $\mathrm{H}$ ) gas that have been observed in absorption in several radio sources with different radio power (e.g., Morganti et al. 1998, 2005b, 2013, 2016; Oosterloo et al. 2000; Mahony et al. 2013; Geréb et al. 2015; Allison et al. 2015). Some of these objects host young or restarted AGN whose central radio source shows characteristics of a compact steep spectrum (CSS) object. This provides valuable insight into the evolution of radio galaxies, because CSS sources are considered to be the younger counterparts of the much larger Fanaroff-Riley-type radio galaxies (e.g., O'Dea 1998; Kunert-Bajraszewska et al. 2010; Orienti 2016). The radio continuum is commonly a few kiloparsec or smaller in size, which limits the spatial scales on which the $\mathrm{H}_{\mathrm{I}}$ outflow can be observed. In the two radio galaxies 3C 305 and 3C 295, the outflows were found to be on kiloparsec scales (Morganti et al. 2005a; Mahony et al. 2013). However, in most cases, sub-arcsecond angular resolution is needed in order to locate the outflow and trace its structure.

The angular resolution can be achieved by very long baseline interferometry (VLBI), which has been used to study the associated $\mathrm{HI}_{\mathrm{I}}$ gas in absorption in various radio sources (e.g., Carilli et al. 1998; Peck et al. 1999; Peck \& Taylor 2001; Vermeulen et al. 2003, 2006; Struve \& Conway 2010, 2012; 
Araya et al. 2010). The first detection of a broad H I outflow with VLBI was reported by Oosterloo et al. (2000) in the Seyfert 2 galaxy IC 5063. Of particular relevance for our study was the successful imaging and mapping of the $\mathrm{H}$ I outflow in the young restarted radio galaxy 4C 12.50 by Morganti et al. (2013). The broad bandwidth and high sensitivity of the VLBI observation revealed the outflow in this source as an extended cloud cospatial with the southern extent of the radio continuum emission. This is offset to the H I gas at the systemic velocity, which is located north of the nucleus. The study determined a mass of the cloud of up to $10^{5}$ solar masses $\left(M_{\odot}\right)$ and a mass outflow rate of $16-29 M_{\odot} \mathrm{yr}^{-1}$. A comparison with an unresolved $\mathrm{H}_{\mathrm{I}}$ absorption spectrum obtained with the Westerbork Synthesis Radio Telescope (WSRT) showed that the entire absorption was recovered by VLBI. The results provide the strongest evidence for this type of radio AGN so far that the $\mathrm{H}$ I outflow is driven by the jet.

Based on these results, we performed VLBI observations of $\mathrm{H} \mathrm{I}$ in absorption of a small sample comprising 3C 236, 3C 293, and 4C 52.37. This initial work will pave the way for future VLBI observations of $\mathrm{H}$ I gas in a larger sample selected from the WSRT H I absorption survey (Geréb et al. 2015; Maccagni et al. 2017).

In this paper, we focus on 3C 236 at a redshift of $z=0.1005$ (Hill et al. 1996), which is one of the largest known radio galaxies extending about 4.5 Mpc (Willis et al. 1974; Barthel et al. 1985; Schilizzi et al. 2001). This source represents a restarted AGN, i.e., it exhibits signs of different stages of AGN activity. The large-scale morphology (top panel in Fig. 1) stems from a previous cycle of activity compared to the CSS-type radio source in its inner $2 \mathrm{kpc}$ region, which is the result of the most recent cycle. The inner radio emission has a dynamical timescale consistent with the age of the young star formation region (O'Dea et al. 2001; Schilizzi et al. 2001; Tremblay et al. 2010).

The host galaxy of 3C 236 features a large outer and a smaller inner dust lane, which are slightly offset in position angle (PA) with respect to each other (O'Dea et al. 2001; Schilizzi et al. 2001; Labiano et al. 2013). The inner dust lane has a PA of $\sim 30^{\circ}$ that is almost perpendicular to the subkiloparsec scale radio jet. VLBI observations by Schilizzi et al. (2001) showed that the jet is oriented in northwest direction extending from the brightest feature, which is synchrotron selfabsorbed and thus likely to be the core region. The southeast lobe produced by the counter jet is positionally coincident with parts of the inner dust lane, and its morphology is considered to be partly a result of jet-ISM interaction. The background image in the middle panel of Fig. 1 shows a zoom-in of the inner dust lane overlaid by the brightness distribution of the radio source as obtained in this paper (bottom panel of Fig. 1, see Sect. 3).

Low-resolution H I absorption spectra reveal a deep narrow absorption feature near the systemic velocity (van Gorkom et al. 1989) and a broad (up to $1000 \mathrm{~km} \mathrm{~s}^{-1}$ ) shallow blue wing corresponding to a mass outflow rate of $\sim 47 M_{\odot} \mathrm{yr}^{-1}$ (Morganti et al. 2005b). Based on H I VLBI observations by Struve \& Conway (2012), the narrow component has been interpreted as H I gas located in a regular rotating disk that is cospatial to the southeast lobe about 250 mas from the nucleus. Because of bandwidth limitations, the VLBI data were not able to cover the velocity range of the outflow.

The optical AGN has been classified as a low-excitation radio galaxy (LERG, Buttiglione et al. 2010; Best \& Heckman 2012), which makes it less likely that strong quasar or starbustdriven winds are the origin of the outflow, but rather the jets. There are also signs of an outflow of ionized gas (Labiano et al. 2013). The cold (CO) and warm $\left(\mathrm{H}_{2}\right)$ molecular gas have only
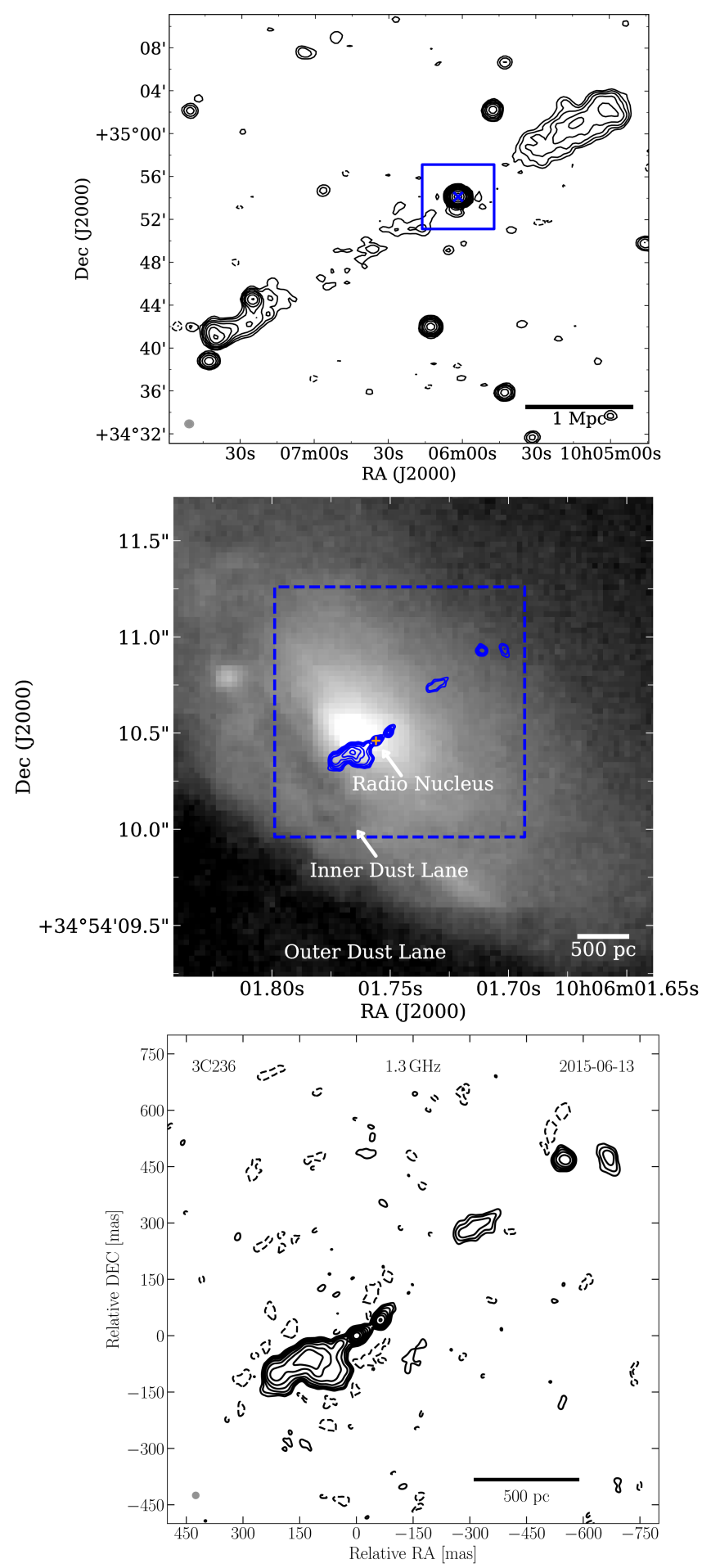

Fig. 1. Left panel: $1.4 \mathrm{GHz}$ VLA NVSS image of the large-scale radio emission of 3C 236 (Condon et al. 1998). The blue square and cross highlight the area covered by our VLA observation and the pointing of the VLBI observation, respectively. Middle panel: graycolored background shows a zoom-in into an archival Hubble Space Telescope $V$-band image (ACS/HRC/F555W, O'Dea et al. 2001). The blue contour lines trace the VLBI radio continuum emission, starting for visibility at $5 \times \sigma_{\text {noise, } \mathrm{VLBI}}$. The orange cross marks the position of the VLBI core from Taylor et al. (2001) to which our VLBI image was aligned to in this montage. The dashed lines mark the plot range of the VLBI image shown in the bottom panel. Bottom panel: VLBI image obtained by our observation. The dashed and solid black contour lines trace negative and positive brightness starting from $3 \times \sigma_{\text {noise,VLBI }}$ and increasing logarithmically by a factor of 2 . 
been detected in a disk-like geometry aligned with position angle of the inner dust lane, but the latter has a significant turbulent component (Nesvadba et al. 2011; Labiano et al. 2013).

This paper presents new VLBI observations with a larger bandwidth than previous data to localize the H I outflow with respect to the radio jet and constrain its properties in combination with new lower resolution Very Large Array (VLA) data. It is structured as follows: in Sect. 2 we present the data and subsequent calibration procedure. This is followed by a presentation of our results in Sect. 3 and discussion in Sect. 4. We end the paper with our conclusions and a summary in Sect. 5 .

Throughout this paper, we adopt a standard $\Lambda$ CDMcosmology $\left(H_{0}=70 \mathrm{~km} \mathrm{~s}^{-1} \mathrm{Mpc}^{-1}, \Omega_{\mathrm{m}}=0.3, \Omega_{\lambda}=0.7\right)$ based on which, 1.0 mas corresponds to about $1.8 \mathrm{pc}$ for $3 \mathrm{C} 236$. It is important to point out that a range of values are available for the systemic velocity $v_{\text {sys }}$ (see also Struve \& Conway 2012). Labiano et al. (2013) determined $v_{\text {sys }}^{\mathrm{CO}} \approx 29761 \mathrm{~km} \mathrm{~s}^{-1}$ based on the $\mathrm{CO}$ spectrum, although the spectral setup prohibited sampling of the continuum emission at low velocities, limiting the Gaussian fit to the spectrum. Nevertheless, this value is close to the SDSS value of $v_{\text {sys }}^{\text {SDS }} \approx 29740 \mathrm{~km} \mathrm{~s}^{-1}$. Struve \& Conway (2012) reported a value of $v_{\text {sys }}^{\mathrm{HI}} \approx 29820 \mathrm{~km} \mathrm{~s}^{-1}$ assuming that parts of the observed $\mathrm{H}$ I are constrained within a disk. However, the H I was detected in absorption, which makes it difficult to measure the full extent of the disk (see also Sect. 4.2). Therefore, we use $v_{\text {sys }}^{\mathrm{CO}}$ throughout this paper as a reference value.

\section{Observation and data reduction}

\subsection{VLBI observation}

The source 3C 236 was observed with a global VLBI array of 14 telescopes on 2015 June 13 (project code: GN002B). The observational setup is summarized in Table 1. The array included the full VLBA and stations from the European VLBI Network (EVN). In addition, Arecibo (Puerto Rico) participated for $2 \mathrm{~h}$. The observation lasted a total of $15 \mathrm{~h}$, with the EVN and VLBA observing for $\sim 11 \mathrm{~h}$ and $\sim 12 \mathrm{~h}$, respectively, and it included an overlap of the two arrays of $\sim 8 \mathrm{~h}$. The stations at Onsala and Kitt Peak (VLBA) were flagged during the calibration due to unusually high system temperatures and bandpass problems, respectively. The data were correlated at the Joint Institute for VLBI ERIC (JIVE), providing two data sets: one with 4 IFs each with 32 channels ("continuum pass"), and one with 1 IF with 512 channels ("spectral line pass"). The source $0958+346$ (BZQ J1001 + 3424) was observed as the phase reference calibrator, while $0955+326(3 \mathrm{C} 232)$ and $0923+392$ ([HB89] $0923+392$ ) served as the bandpass calibrators.

The data were calibrated in two steps using standard procedures in the Astronomical Image Processing Software (AIPS, version 31DEC15) package (Associated Universities 1999), beginning with the data from the continuum pass. The amplitude calibration and initial flagging were provided by the EVN pipeline. As a next step, manual phase calibration on a single scan of the calibrator was performed to remove the instrumental delay. This was followed by a global fringe fit of the calibrators to correct for the phase delay and rate with the solutions applied to the target source. Finally, the bandpass was corrected using the bandpass calibrators, and the solutions were applied to the phase reference and target source. For the spectral line pass, the amplitude calibration and initial flagging was also taken from the EVN pipeline. The phase calibration was performed using the solutions from the manual phase calibration and global fringe fit of the continuum pass, which was followed by the bandpass calibration. Afterward, the data were separated into a data cube with full spectral resolution and a continuum data set with all channels averaged together.

The continuum data were further processed in DIFMAP (Shepherd et al. 1994; Shepherd 2011). This entailed imaging of the brightness distribution of the source using the CLEAN algorithm (Högbom 1974) in combination with phase self-calibration and flagging of corrupted visibilities. When a sufficiently good model was found, a time-independent gain correction factor was determined for each telescope through amplitude self-calibration. The iterative process of imaging and phase self-calibration with subsequent time-dependent amplitude self-calibration was repeated several times with a decreasing solution interval for amplitude self-calibration.

The resulting continuum image was used to perform a single phase self-calibration of the data cube with full spectral resolution in AIPS. After carefully inspecting the channels and flagging of corrupted visibilities, the continuum was subtracted in the visibility domain using a linear fit to the first and last 100 channels (AIPS task UVLIN). Since we focus on the faint and broad component of the absorption, we averaged over three consecutive channels to improve the sensitivity. The data were corrected for the Doppler shift in frequency caused by the rotation and movement of the Earth. Finally, a redshift correction was applied to the channel width in observed frequency to convert into rest-frame velocity following Meyer et al. (2017).

Each channel of the spectral line cube was imaged individually with robust weighting set to 1 and a $(u, v)$-taper of $10 \mathrm{M} \lambda$ to further improve the sensitivity. We found that this tapering of the visibility data provides the best combination of resolution and sensitivity. It is similar to Struve \& Conway (2012). The channels were only imaged if significant negative flux density was found in the area covered by continuum emission. The resulting cube covers a velocity range of $28117-31209 \mathrm{~km} \mathrm{~s}^{-1}$ at a channel resolution of $21.7 \mathrm{~km} \mathrm{~s}^{-1}$, which is effectively doubled to $43.4 \mathrm{~km} \mathrm{~s}^{-1}$ through Hanning-smoothing.

In order to compare the image cube and the continuum image, both were restored with the same circular restoring beam of 20 mas. This is close to the synthesized beam due to the $(u, v)$ tapering and similar to Struve \& Conway (2012). The noise levels were determined by fitting a Gaussian distribution to the pixels that were not contaminated by emission from the target, following the procedure outlined in Böck (2012). For the subsequent analysis, the average noise level of the cube was used as a reference value. An overview of the image parameters is given in Table 2. The overall amplitude calibration uncertainty of the VLBI data was estimated to be about $15 \%$ based on multiple iterations of imaging and self-calibration of the continuum data. The total flux density was measured in the image plane using the CASA Viewer (International Consortium Of Scientists 2011). We estimated the uncertainty of the peak and total flux density measurement as $\sqrt{\left(N_{\text {beam }} \times \sigma_{\text {noise }}\right)^{2}+\left(0.15 \times S_{\text {tot }}\right)^{2}}$ following Nyland et al. (2016), where $N_{\text {beam }}$ corresponds to the number of beams covered by the source. However, we note that in our case, the first term has only a marginal effect on the uncertainty.

\subsection{VLA observation}

The VLA observed 3C 236 for 40 min in A-array configuration (project code: 11A-166) on 2011 August 10. The setup is summarized in Table 1. The source $1328+307$ (3C 286) was used as the flux density calibrator and $1015+359(\mathrm{~B} 21015+35 \mathrm{~B})$ as the complex gain calibrator. 
Table 1. Observation properties.

\begin{tabular}{|c|c|c|c|c|c|c|c|c|c|c|}
\hline Array $^{a}$ & $\operatorname{Code}^{b}$ & Date & $\begin{array}{c}v_{\mathrm{obs}}^{c} \\
(\mathrm{GHz})\end{array}$ & $\begin{array}{c}T_{\mathrm{obs}}^{d} \\
(\mathrm{~min})\end{array}$ & Pol. $^{e}$ & Correlator pass & $\begin{array}{c}\text { IFs } \\
(\mathrm{MHz})\end{array}$ & $\mathrm{BW}^{f}$ & $\begin{array}{c}N_{\mathrm{ch}}^{g} \\
(\mathrm{kHz})\end{array}$ & $\Delta v^{h}$ \\
\hline \multirow{2}{*}{$\mathrm{EVN}+\mathrm{VLBA}+\mathrm{Ar}$} & \multirow{2}{*}{ GN002B } & \multirow{2}{*}{ 2015-06-13 } & \multirow{2}{*}{1.293} & \multirow{2}{*}{500} & \multirow{2}{*}{ Dual } & continuum & 4 & 16 & 32 & 500 \\
\hline & & & & & & Spectral line & 1 & 16 & 512 & 31.25 \\
\hline VLA & $11 \mathrm{~A}-166$ & 2011-08-10 & 1.283 & 40 & Dual & Spectral line & 1 & 16 & 256 & 62.5 \\
\hline
\end{tabular}

Notes. ${ }^{(a)}$ Array used for observation. EVN: Effelsberg (Germany), phased-up Westerbork ( 5 stations, Netherlands), Jodrell-Bank (United Kingdom), Onsala (Sweden); VLBA (USA): Los Alamos (NM), Kitt Peak (AZ), St. Croix (VI), Mauna Kea (HI), Hancock (NH), Brewster (WA), Fort Davis (TX), North Liberty (IA), Pie Town (NM), Owens Valley (CA); Ar: Arecibo (Puerto Rico). ${ }^{(b)}$ Experiment code, ${ }^{(c)}$ observing frequency, (d) observing time. For the VLBI experiment, this represents the total on-source time of the whole array. ${ }^{(e)}$ Polarization: dual means that two polarization were used (LL and RR), ${ }^{(f)}$ bandwidth (of each IF), ${ }^{(g)}$ number of channels in a single band or IF, ${ }^{(h)}$ channel width in frequency.

Table 2. Image properties.

\begin{tabular}{|c|c|c|c|c|c|c|}
\hline Data & $\begin{array}{c}\sigma_{\text {noise }}^{a} \\
\left(\mathrm{mJy} \mathrm{beam}^{-1} \mathrm{ch}^{-1}\right)\end{array}$ & Beam $^{b}$ & $\begin{array}{c}\Delta v^{c} \\
\left(\mathrm{~km} \mathrm{~s}^{-1}\right)\end{array}$ & $N_{\mathrm{ch}}^{d}$ & $\begin{array}{c}S_{\text {peak }}^{e} \\
\left(\mathrm{Jy} \mathrm{beam}^{-1}\right)\end{array}$ & $\begin{array}{l}S_{\text {tot }}^{f} \\
(\mathrm{Jy})\end{array}$ \\
\hline \multicolumn{7}{|c|}{ VLBI } \\
\hline Continuum & 0.23 & 20 mas $\times 20$ mas & - & 1 & $0.145 \pm 0.020$ & $1.35 \pm 0.20$ \\
\hline Cube & 0.37 & $20 \mathrm{mas} \times 20 \mathrm{mas}$ & $21.7(43.4)$ & 143 & - & - \\
\hline \multicolumn{7}{|c|}{ VLA } \\
\hline Continuum & 2.1 & $1^{\prime \prime} .7 \times 1^{\prime \prime} .2,-67^{\circ}$ & - & 1 & $2.883 \pm 0.086$ & $3.37 \pm 0.10$ \\
\hline Cube (VLBI) & 1.1 & $2^{\prime \prime} .0 \times 1^{\prime \prime} .5,-81^{\circ}$ & $21.7(43.4)$ & 157 & - & - \\
\hline Cube (WSRT) & 1.1 & $2^{\prime \prime} .0 \times 1^{\prime \prime} .5,-81^{\circ}$ & $20.0(40.0)$ & 186 & - & - \\
\hline
\end{tabular}

Notes. ${ }^{(a)}$ Noise level. For the cubes, this value represents the average value over all channels. ${ }^{(b)}$ Synthesized beam as major axis, minor axis, and the position angle. ${ }^{(c)}$ Channel width in velocity of the cubes after re-sampling. The value in brackets corresponds to the effective resolution after Hanning-smoothing. ${ }^{(d)}$ Number of channels of the cubes. ${ }^{(e)}$ Total flux density of the continuum image. ${ }^{(f)}$ Peak flux density of the continuum image.

The data were processed with MIRIAD (Sault et al. 1995, 2011). Because of technical difficulties, one polarization was lost and had to be flagged completely. The data were calibrated using standard procedures for the VLA. A continuum image was produced using CLEAN and self-calibration. The spectral-line data were continuum subtracted using only channels devoid of the broad absorption. Further processing was performed in AIPS. The VLA cube was averaged to match the velocity resolution of the VLBI data, cleaned in the region of the continuum emission, and then it was Hanning smoothed. Consistent with the VLBI spectral line data, the channel width was redshift-corrected to the rest-frame velocity width. The resulting image cube covers a velocity range from 28316 to $31706 \mathrm{~km} \mathrm{~s}^{-1}$. For a comparison with the WSRT spectrum presented in Morganti et al. (2005b), we also created a second cube that matches the velocity resolution of the WSRT data. The parameters of the continuum image and the cube are given in Table 2. We estimate that the uncertainty of the absolute flux density scale at this frequency and given the calibrators is $\sim 3 \%$ based on Perley \& Butler (2013).

\section{Results}

\subsection{VLA and VLBI continuum}

The VLA continuum image covers the central $6^{\prime} \times 6^{\prime}$ $(650 \mathrm{kpc} \times 650 \mathrm{kpc})$ of $3 \mathrm{C} 236$ (see Fig. 1). The recovered radio emission is unresolved and yields a flux density of about $3.37 \pm 0.10 \mathrm{Jy}$. No further extended emission is detected. The flux density is consistent with the value of $3.324 \pm 0.097 \mathrm{Jy}$ obtained from the central region of the radio emission by the lower-resolution NVSS survey at $1.4 \mathrm{GHz}$ (Condon et al. 1998). The full VLBI continuum emission of 3C 236 from our observation is shown in the bottom panel of Fig. 1. The total flux density is about $1.35 \pm 0.20 \mathrm{Jy}$, which is consistent with the value obtained by Struve \& Conway $(2012 ; 1.36 \mathrm{Jy})$. It corresponds to about $40 \%$ of the flux density measured by the VLA observation. The source is significantly extended, covering approximately $1^{\prime \prime}(\sim 1.8 \mathrm{kpc})$, but most of the radio emission is localized within 400 mas $(\sim 720 \mathrm{pc})$.

The difference in flux density between VLBI and VLA of about 2 Jy can have different reasons. First, the shortest baseline of the VLBI array limits the largest angular scale on which emission can be recovered to about 600 mas. Any extended emission on larger scales is resolved out by the interferometer. However, there could still be a significant amount of emission within the largest angular scale limit. Assuming the emission is uniformly distributed over the entire area, then an integrated flux density of more than $600 \mathrm{mJy}$ is necessary to achieve a brightness at the $3 \sigma_{\mathrm{VLBI}}$,cont sensitivity limit of the VLBI image. Second, the consistency between our VLA and the NVSS flux density shows that all of the undetected emission must be within the area covered by the synthesized beam of the VLA observation, that is, $1^{\prime \prime} \cdot 7 \times 1{ }^{\prime \prime}$. $(3.1 \mathrm{kpc} \times 2.2 \mathrm{kpc})$. Assuming again a uniform distribution of the undetected emission over this region leads to a brightness of about $0.6 \mathrm{mJy}_{\text {beam }}{ }^{-1}$. This is just below the $3 \sigma_{\mathrm{VLBI}}$,cont - limit. Thus, a global VLBI experiment including the VLA and eMERLIN would provide the sensitivity and short spacing to recover the whole emission.

The morphology is overall consistent with previous VLBI observations by Schilizzi et al. (2001) and Struve \& Conway (2012). The sensitivity of our VLBI image is about a factor of three better than the image from Struve \& Conway (2012), but we do not detect significantly more extended emission or any movement of features in the jet at the given resolution. Following 
Schilizzi et al. (2001), we consider the location of the VLBI core region to coincide with the brightest feature at the phase center of the image and the jet to extend to the northwest. The emission southeast of the core region corresponds to the radio lobe created by the counter jet. Because of the chosen restoring beam, the brightest feature is a blend of the emission from the VLBI core and part of the innermost jet. We refer to this as the nuclear region from here on. In the following, we continue to focus on the inner most 400 mas of the source where the bulk of the radio emission is located. We determine the position angle of the jet as the angle along which the brightest features of the southeast jet are best aligned on to be about $116^{\circ}$, which is consistent with the measurement of Struve \& Conway (2012) of $117^{\circ}$.

\section{2. $H_{1}$ absorption spectrum}

Figure 2 (top panel) shows the unresolved VLA spectrum between $28500 \mathrm{~km} \mathrm{~s}^{-1}$ and $30900 \mathrm{~km} \mathrm{~s}^{-1}$ in combination with the WSRTspectrum from Morganti et al. (2005b). The spectra taken with both instruments are consistent and show the same features: a deep and narrow absorption that smoothly falls off toward higher velocities, but has a complex, broad wing toward lower velocities. The consistency between the two spectra implies that all of the absorption stems from scales smaller than the beam size of the VLA.

Two spatially integrated VLBI spectra are shown in the bottom panel of Fig. 2 between $29000 \mathrm{~km} \mathrm{~s}^{-1}$ and $30400 \mathrm{~km} \mathrm{~s}^{-1}$. They were compiled by considering those pixels in the image cube that are located within the region marked by the $3 \sigma_{\mathrm{VLBI}, \text { cont }}$ contour line of the continuum image. They differ in terms of the selection of pixels in the cube. For the dashed blue line labeled "VLBI (clipped)", a very conservative limit of $\left|S_{\text {pixel,cube }}\right| \geq 3 \times \sigma_{\text {VLBI,cube }}$ was used, where $\left|S_{\text {pixel,cube }}\right|$ is the absolute value of the pixel brightness. No such limit was applied for the compilation of the spectrum marked by the solid blue line(labeled "VLBI") and the VLA spectrum (solid red line), which is unresolved, in contrast to the VLBI spectrum.

The VLBI without clipping shows a deep and narrow absorption feature consistent with the previous measurement by Struve \& Conway (2012), but its depth and width do not match the VLA reference spectrum. Because the deep absorption is likely due to gas associated with the extended dust lane (see Sect. 3.3), the undetected absorption flux density is likely related to structure resolved out as seen from the missing continuum flux density. However, some unsettled gas appears in our VLBI observations (see Sect. 3.3). More interestingly, the observations reveal some of the outflowing gas (bottom panel of Fig. 2). However, the VLBI observation recover only a small fraction of the blueshifted wing of the H i profiles. We discuss the possible implications of this for the distribution of the outflowing gas in Sect. 4.1.

\subsection{H। gas distribution}

The spatial distribution of the HI gas is shown in the central panel of Fig. 3. It shows the optical depth $\tau$ integrated over the same velocity range as in the bottom panel of Fig. 2, that is, between $29000 \mathrm{~km} \mathrm{~s}^{-1}$ and $30400 \mathrm{~km} \mathrm{~s}^{-1}$. The optical depth is defined as $\tau=\log \left(1-\Delta S_{\text {abs }} /\left(c_{\mathrm{f}} S_{\text {cont }}\right)\right)$, where $\Delta S_{\text {abs }}$ and $S_{\text {cont }}$ correspond to the absorbed and the continuum flux density, respectively, and the covering factor $c_{\mathrm{f}}$ is assumed to be unity. In order to avoid integrating over noise and to obtain a reliable if conservative distribution of the $\mathrm{H}$ I gas, we take into
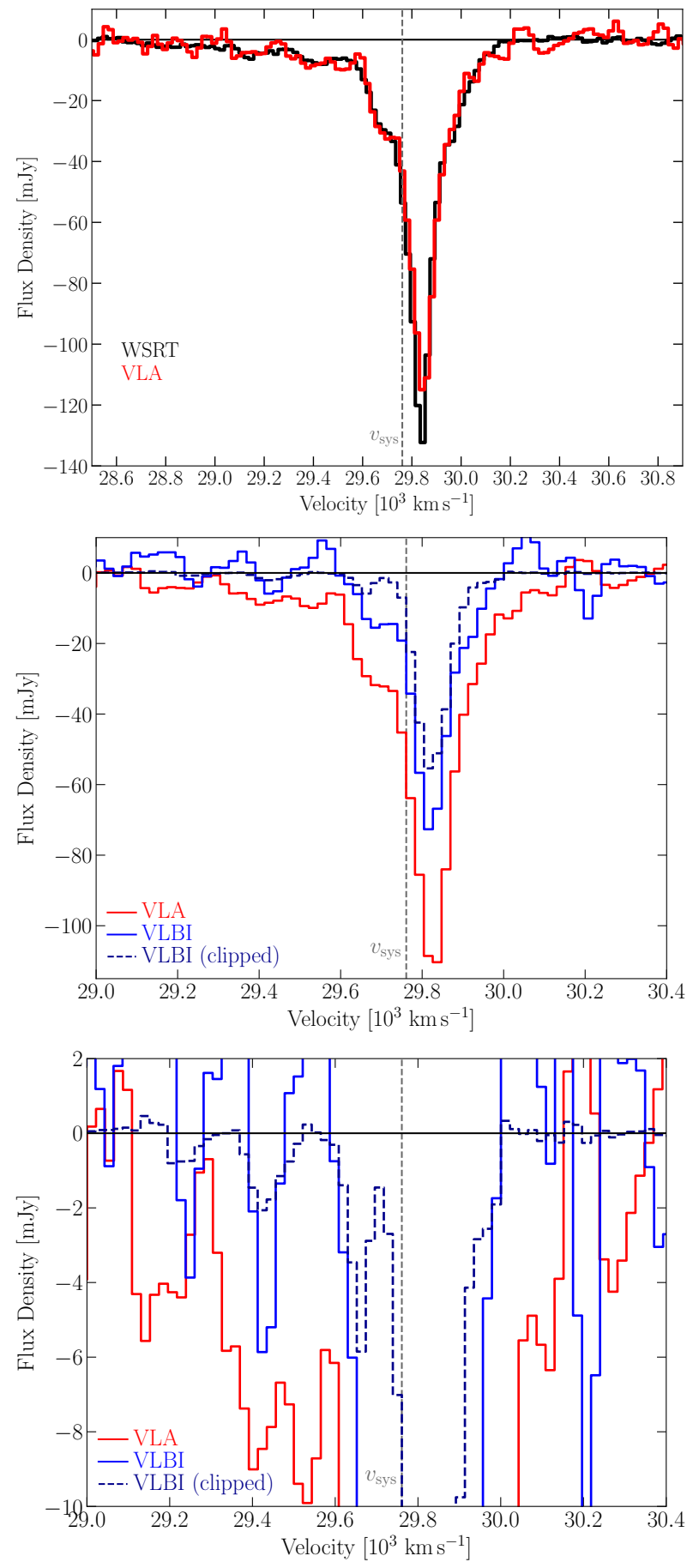

Fig. 2. H I absorption spectra of 3C 236. The dashed vertical line marks the systemic velocity from Labiano et al. (2013). Top panel: WSRT (black) spectrum from Morganti et al. (2005b) and VLA (red) spectrum between 28500 and $30900 \mathrm{~km} \mathrm{~s}^{-1}$. Here, the velocity resolution of the VLA spectrum was matched to the WSRT data. Middle panel: spatially integrated VLBI with (dark blue, dashed) and without (blue, solid) clipping of the cube pixels at the $3 \sigma_{\text {VLBI,Cube }}$ between 29000 and $30400 \mathrm{~km} \mathrm{~s}^{-1}$. For the VLA spectrum (red), the spectral resolution was matched to the VLBI spectrum. Bottom panel: same as the middle panel, but zoomed-in in flux density.

account only channels with $\leq-3 \sigma_{\mathrm{VLBI}, \text { cube }}$. In addition to the integrated optial depth $\int \tau \mathrm{d} v$, this figure shows single-pixel spectra of optical depth extracted at specific locations of the radio continuum, for which the detection limit of channels was not applied. 

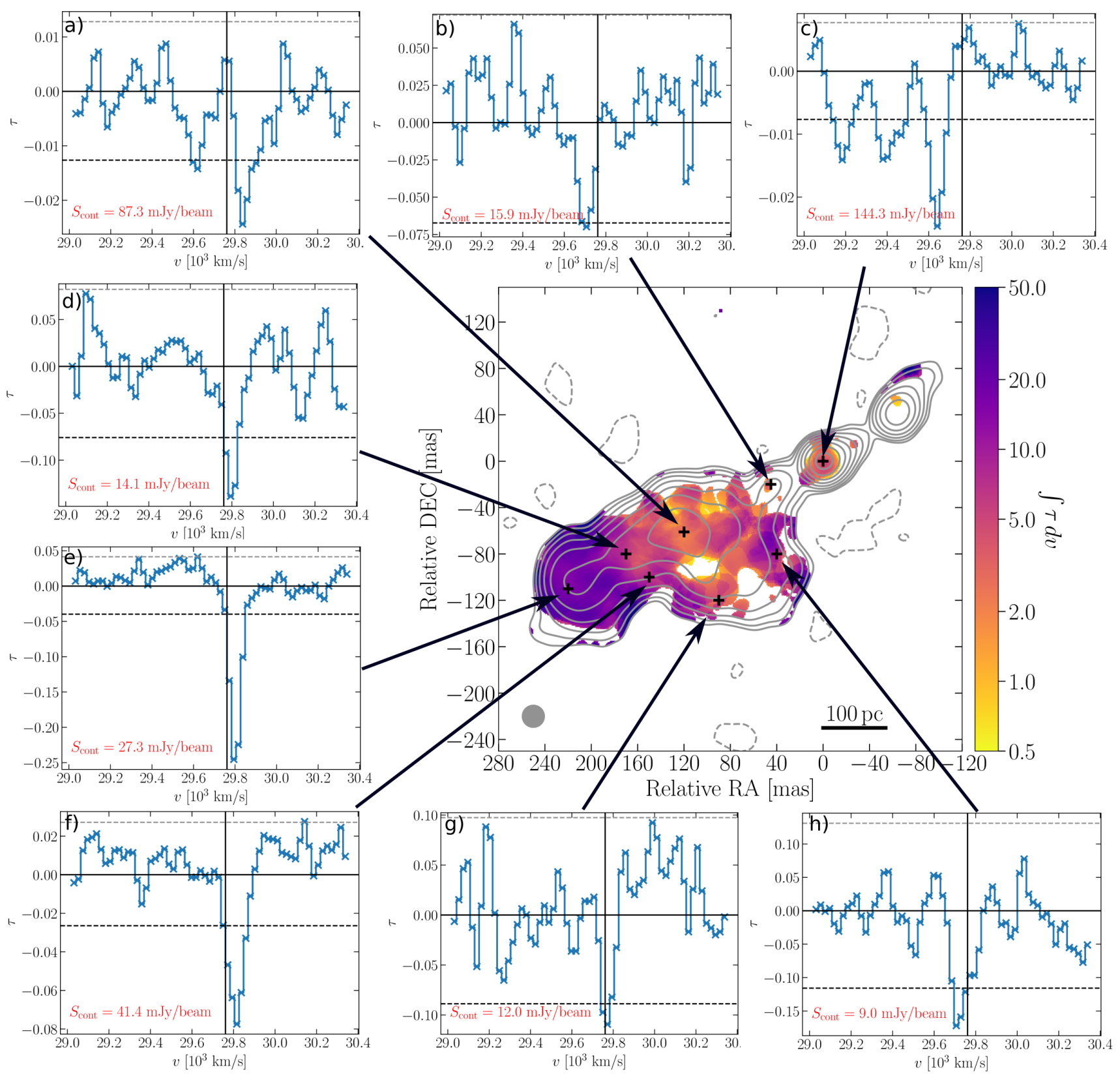

Fig. 3. Central panel: continuum image of the central region of $3 \mathrm{C} 236$ shown in Fig. 1 . The contour lines start at $3 \sigma_{\mathrm{VLBI}, \mathrm{cont}}$ and increase logarithmically by a factor of 2 . Solid and dashed contour lines correspond to positive and negative flux density, respectively. The black crosses mark areas where the spectrum was extracted between $29000-30000 \mathrm{~km} \mathrm{~s}^{-1}$. Outer panels: optical depth spectrum shown as the ratio of the absorbed flux density to the continuum flux density. The dashed line represents the optical depth detection limit at the $3 \sigma_{\mathrm{VLBI}, \text { cube }}$ noise level. The solid vertical line marks the systemic velocity.

The map of $\int \tau \mathrm{d} v$ reveals a complex gas distribution across the southeast lobe and compact absorption toward the nucleus. In particular, the gas covering the southeast lobe exhibits significant changes in $\int \tau \mathrm{d} v$. In the central and brightest part of the lobe, $\int \tau \mathrm{d} v$ reaches its lowest values, but there are several gaps in the gas distribution, in particular toward the nuclear region. The highest values of $\int \tau \mathrm{d} v$ are measured toward the end of the southeast lobe. In this region the majority of the H I gas is located that leads to the narrow, deep feature in the integrated spectrum.

The spectra of $\tau$ in Figs. 3a-h show the range of optical depth probed by our observation. The lowest optical depth is reached toward the nuclear region as it has the brightest part of the radio source (Fig. 3c). Here, we are sensitive down to $\tau \gtrsim 0.0077$ at the $3 \sigma_{\mathrm{VLBI}, \mathrm{cube}}$ noise level and find three distinct kinematic features that are discussed in greater detail later in this section. Toward the southeast lobe, the optical depth sensitivity varies. The lowest optical depth is reached in the central region of the lobe (Fig. 3a) with $\tau \gtrsim 0.013$, where we also find a more complex kinematic structure than in the other regions.

In order to investigate the spatial and velocity distribution of these features in greater detail, we show position-velocity plots along different position angles in Figs. 4a-e. Again, we focus on the velocity range of $29000 \mathrm{~km} \mathrm{~s}^{-1}$ and $30400 \mathrm{~km} \mathrm{~s}^{-1}$. For comparison, the central panel shows the same map of $\int \tau \mathrm{d} v$ as in Fig. 3. 

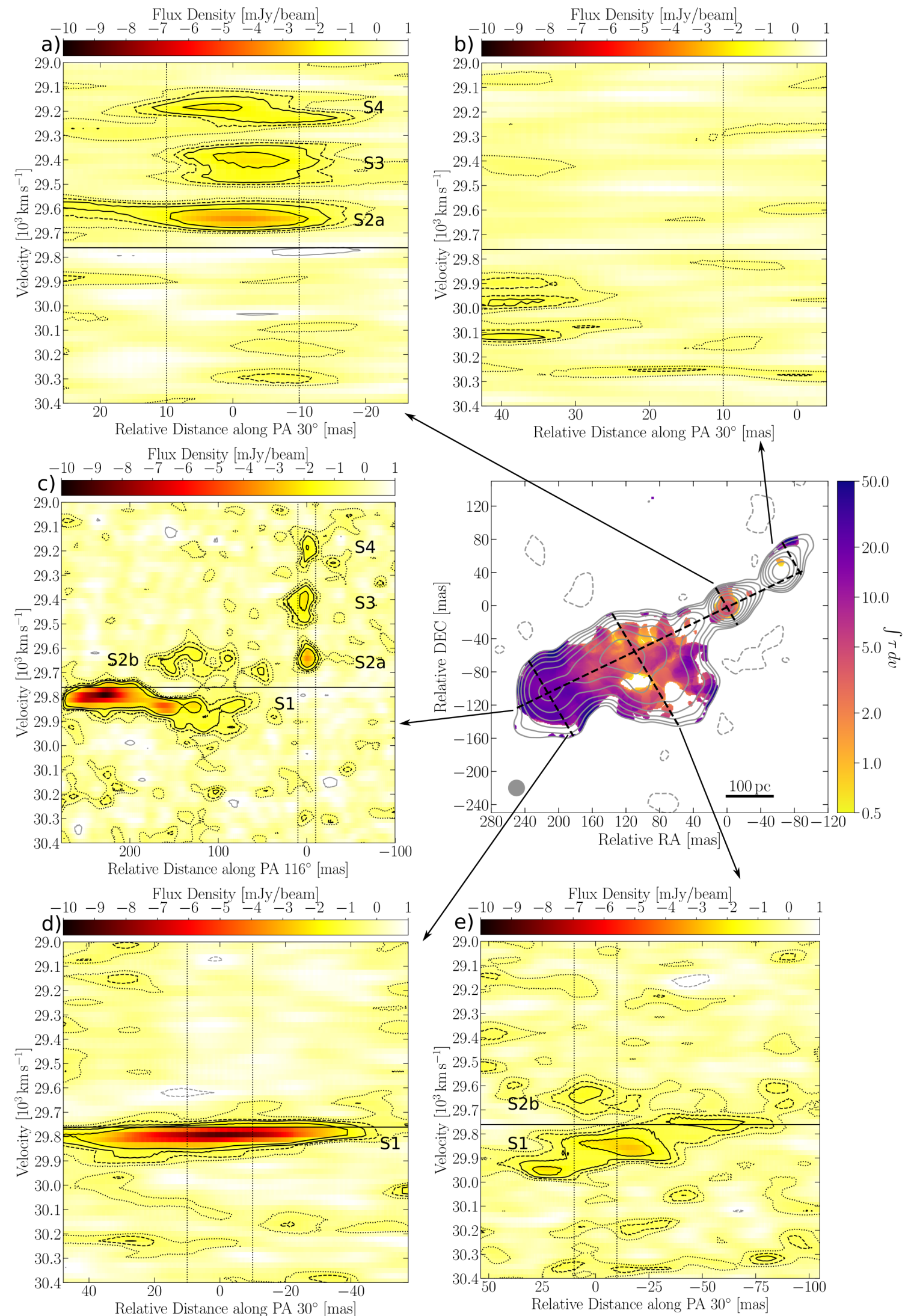

Fig. 4. Position-velocity plots (panels $a-e$ ) from 29000 to $30400 \mathrm{~km} \mathrm{~s}^{-1}$ for slices along different position angles. The same color scale is applied to all position-velocity plots. The black dashed vertical lines mark the size of the synthesized beam for comparison, and the black solid horizontal line refers to the systemic velocity from Labiano et al. (2013). The black contour lines correspond to -1 (dotted lines), -2 (dashed lines), -3 and -5 (solid lines) times $\sigma_{\mathrm{VLBI}, \text { cube }}$, and the solid gray contour line is set to $3 \sigma_{\mathrm{VLBI}, \mathrm{cube}}$. The central panel shows the slices and the optical depth integrated over the same velocity range as the position-velocity plots, but including only channels with $\leq-3 \sigma_{\mathrm{VLBI}, \text { cube }}$. 
Figures $4 \mathrm{c}$ and $\mathrm{d}$ show the velocity structure of the gas along the jet position angle and along the position angle of the inner dust lane, respectively. In particular, Fig. 4d reveals a gradient in velocity similar to Struve \& Conway (2012), which has been interpreted as a signature of an $\mathrm{H}$ I disk aligned with the inner dust lane. Our new data show that this gradient is even more prominent across the central part of the lobe (Fig. 4e). At the $-2 \sigma_{\text {VLBI,cube }}$ level, the gradient would cover almost $300 \mathrm{~km} \mathrm{~s}^{-1}$. We label the disk-related feature in the following S1.

However, more interesting and relevant from these observations are structures that appear to have a disturbed kinematics (see Fig. 4c). S2b is located toward the central part of the lobe and is slightly blueshifted with respect to the peak of the absorption by about $150 \mathrm{~km} \mathrm{~s}^{-1}$. At the $3 \sigma_{\mathrm{VLBI}}$,cube level it is not connected to S1 spatially or in velocity. The clear separation in velocity between $S 2 b$ and $S 1$ could indicate that $S 2 b$ traces a different component of the $\mathrm{H}$ I gas than $\mathrm{S} 1$.

The most important result from the new observations is the finding that the gas cospatial to the nucleus is entirely blueshifted with respect to the peak absorption by up to $\sim 640 \mathrm{~km} \mathrm{~s}^{-1}$ (Figs. 4a,c). The projected size of this region is only about $36 \mathrm{pc}$ or even smaller. It comprises three distinct features labeled S2a, S3, and S4, which are separated in velocity by a few channels. While it is possible that this separation could be due to changes in sensitivity across the channels, we consider this to be the least likely explanation as the variation in sensitivity is not significant. This leaves three other possibilities (see also Sect. 4.1). First, there is no further Hi gas in this region or the optical depth of the gas is too low to be detectable. In this case, the majority of the remaining ouflowing gas would have to be located elsewhere. Second, the cold H I gas clouds could be entrained by warmer H I gas that has a higher spin temperature and thus, a lower optical depth. Third, the gas within the outflow is highly clumpy, which could imply differnces in the covering factor or spin temperature of the gas.

We cannot exclude the possibility of $\mathrm{H}$ I gas cospatial to the northwest part of the jet. Figure $4 \mathrm{~b}$ and the map of $\int \tau \mathrm{d} v$ suggest that there could be gas in this region that is redshifted with respect to the deep absorption. However, these features are just at the $3 \sigma_{\mathrm{VLBI}}$,cube level, very narrow, and generally located at the edge of the continuum emission. Therefore, we cannot consider them reliable detections.

\section{Discussion}

Our VLBI observation has successfully recovered part of the outflowing $\mathrm{H}$ I gas in 3C 236 in the form of distinct, compact clouds (S2a, S3, and S4). They are located primarily cospatial, in projection, to the nuclear region, which has a projected size of $\lesssim 36 \mathrm{pc}$, with one possible exception (S2b). The clouds cover velocities of $150-600 \mathrm{~km} \mathrm{~s}^{-1}$ blueshifted with respect to the H I that is likely related to a rotating disk aligned with inner dust lane (S1). The disk-related gas extends over most over the southeast radio lobe. The overall gas distribution is clumpy, with the majority of the gas concentrated at the end of the lobe. An important characteristic of the H I VLBI data is the significant amount of $\mathrm{HI}$ absorption that is not detected, but inferred from lowangular resolution observations with the VLA and the WSRT.

\subsection{High-velocity HI gas}

As described in Sect. 1, 3C 236 is classified as a LERG, which makes it less likely that the AGN is able to produce powerful radiative winds that could couple to the dust of the galaxy and create strong gaseous outflows. This in turn leaves the radio jet as the main driver for the H I outflow, that is to say, features S2a, S3, $\mathrm{S} 4$, and perhaps S2b. However, we cannot exclude the possibility that S2b is part of the H I disk (see also Sect. 4.2).

The location of S2a, S3, and S4 suggests that the outflow starts already very close to the nucleus of 3C 236 (see also Figs. 5a,b, and c). As these structures are unresolved, we use the restoring beam as an upper limit of the extent of the $\mathrm{H}$ I gas ( $\lesssim 36 \mathrm{pc}$ in projection). In contrast, S2b is extended, and we estimate its projected size to be about $54 \mathrm{pc} \times 15 \mathrm{pc}$.

\subsubsection{Implications of the undected absorption}

There are several implications for the undetected absorbed flux density in our VLBI observation. The diffuse extended continuum emission that is resolved out by the high resolution of VLBI (see Sect. 3.1) limits the area over which we can probe for absorption. However, we could still be able to detect H I absorption in regions outside of the detected continuum emission if the absorption is compact. In some channels, the absorption does extend marginally outside of the $3 \sigma_{\mathrm{VLBI}, \text { cont }}$ contour lines, but is below the $3 \sigma_{\text {VLBI,cube }}$-level.

Another possibility is related to the small fraction of the outflow detected only against the nuclear region. This region is the brightest one with a peak flux density of about $145 \mathrm{mJy}$, which corresponds to an optical depth limit of 0.0026 at the $1 \sigma_{\mathrm{VLBI} \text {,cube }}{ }^{-}$ level or 0.0077 at the $3 \sigma_{\mathrm{VLBI}, \text { cube-level. It is worth noting that }}$ some of the channels between S4 and S3 and between S3 and $\mathrm{S} 2 \mathrm{a}$ have optical depths above the $1 \sigma_{\mathrm{VLBI}}$,cube level at the location of the peak continuum flux density. However, the peak flux density of the lobe is about $87 \mathrm{mJy}$, which corresponds to an optical depth limit of 0.0043 at the $1 \sigma_{\mathrm{VLBI}, \text { cube }}$ level or 0.013 at the $3 \sigma_{\mathrm{VLBI}}$,cube level. The peak optical depth of clouds S3 and S4 is about 0.014 , which is consistent with the $3 \sigma_{\mathrm{VLBI}}$,cube level within our amplitude calibration uncertainty. Thus, we cannot entirely exclude the existence of clouds such as S3 and S4 at larger distances from the nucleus, that is, against the lobe. However, we cannot detect these clouds because of sensitivity limits.

This could be a likely situation and entails that the outflow is indeed more extended, but made up of clouds similar to the one detected against the nucleus. We cannot exclude a component of diffuse gas, but this would also be too faint to be detectable. We can exclude the presence of single clouds producing a depth of the absorption similar to the one detected by the VLA and WSRT (see Fig. 2). Taking the peak flux density of the lobe, the lowest absorption recovered by the WSRT at around $28800 \mathrm{~km} \mathrm{~s}^{-1}$ would correspond to an optical depth of 0.02 . Therefore, such clouds would have been detected at least against the brighter part of the lobe. Neither would we have seen this cloud toward the nuclear region; the peak flux density implies an optical depth of about 0.015 , similar to S3 and S4.

\subsubsection{Properties of the outflowing $\mathrm{H}$ I gas}

Figure 5 shows the column density $N_{\mathrm{H}}$ normalized by the spin temperature $T_{\text {spin }}$ following $N_{\mathrm{HI}} T_{\text {spin }}^{-1} \approx 1.823 \times$ $10^{18} \int \tau(v) \mathrm{d} v \mathrm{~cm}^{-2} \mathrm{~K}^{-1}$, where $v$ is in units of $\mathrm{km} \mathrm{s}^{-1}$. Again, we focus on the velocity range of $\sim 29000 \mathrm{~km} \mathrm{~s}^{-1}$ to $\sim 30400 \mathrm{~km} \mathrm{~s}^{-1}$,

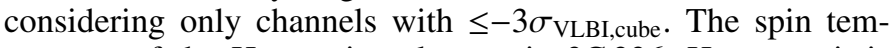
perature of the HI gas is unknown in 3C 236 . However, it is very likely that it reaches higher values in the nuclear region than in the southeast lobe. A similar argument was made by Morganti et al. (2005a), and a $T_{\text {spin }}$ of $\sim 1000 \mathrm{~K}$ is often 
used for gas close to the active nucleus or gas with extremely disturbed kinematics, although measurements of this quantity are scarce (see Morganti et al. 2016; Holt et al. 2006). Thus, it seems reasonable to assume $T_{\text {spin }}=1000 \mathrm{~K}$ for S2a, S3, and S4 and $T_{\text {spin }}=100 \mathrm{~K}$ for $\mathrm{S} 2 \mathrm{~b}$. This results in column densities of $1.8-4.0 \times 10^{21} \mathrm{~cm}^{-2}$ for the clouds cospatial to the nucleus and $1.8 \times 10^{20} \mathrm{~cm}^{-2}$ for $\mathrm{S} 2 \mathrm{~b}$ (see Table 3 ).

We can further estimate the mass outflow rate $\dot{M}_{\mathrm{HI}}$ following Heckman (2002),

$\dot{M}_{\mathrm{HI}} \sim 30 \frac{r_{\star}}{\mathrm{kpc}} \frac{N_{\mathrm{HI}}}{10^{21} \mathrm{~cm}^{-2}} \frac{v}{300 \mathrm{~km} \mathrm{~s}^{-1}} \frac{\Omega}{4 \pi} M_{\odot} \mathrm{yr}^{-1}$,

where $r_{\star}$ is the distance of the cloud, $v$ is its velocity, and $\Omega$ is its solid angle, which is assumed to be $\pi$. We estimate $v$ for each of the features relative to the peak velocity of S1 (see Fig. 4c) and assume that $r_{\star}$ is given by the size of the beam. The only exception is $\mathrm{S} 2 \mathrm{~b}$, for which we measure a distance of $\sim 150$ mas in Fig. 4c, leading to $r_{\star} \sim 310 \mathrm{kpc}$ (deprojected). The resulting values are listed in Table 3 . As both $N_{\mathrm{H}}$ and $r_{\star}$ are lower limits, the actual mass outflow produced by these features is likely to be higher. Because of the order-of-magnitude lower column density compared to the clouds cospatial to the nucleus, S2b has the lowest value. This is also the case for the density of all four clouds, which ranges from $30-50 \mathrm{~cm}^{-3}$ for $\mathrm{S} 2 \mathrm{a}, \mathrm{S} 3$, and $\mathrm{S} 4$ compared to $2 \mathrm{~cm}^{-3}$ for S2b (Table 3). For the former clouds, a spherical geometry was assumed, and for the latter, an ellipsoidal geometry.

Morganti et al. (2005b) estimated the total mass outflow rate to be $\sim 47 M_{\odot} \mathrm{yr}^{-1}$ based on the full extent of the unresolved $\mathrm{H}$ I absorption spectrum $\left(v \sim 1500 \mathrm{~km} \mathrm{~s}^{-1}\right)$ and assuming an homogeneous distribution of the gas over a radius of $0.5 \mathrm{kpc}$. Thus, we consider this value an upper limit on the total mass outflow rate. Integrating over $\mathrm{S} 2 \mathrm{a}, \mathrm{S} 3$, and $\mathrm{S} 4$ yields $N_{\mathrm{H}} \sim 7.8 \times$ $10^{21} \mathrm{~cm}^{-2}$ and $\dot{M}_{\mathrm{HI}} \sim 5 M_{\odot} \mathrm{yr}^{-1}$. This suggests that at least $10 \%$ of the estimated total mass outflow rate is located close (in projection) to the nucleus.

Following Holt et al. (2006), the kinetic energy of an outflow is given by

$E_{\mathrm{kin}} \approx 6.34 \times 10^{35} \frac{\dot{M}}{2}\left(v_{\mathrm{out}}^{2}+\frac{F W H M^{2}}{1.85}\right)$,

where FWHM is the full width at half-maximum of the line. Labiano et al. (2013) fitted three Gaussian distributions to the H I WSRT spectrum. One of the distributions described the broad, blue wing of the spectrum with the following parameters: $v_{\text {hel }}=29474 \mathrm{~km} \mathrm{~s}^{-1}$ and $F W H M \sim 1100 \mathrm{~km} \mathrm{~s}^{-1}$. Using these values and $\dot{M}_{\mathrm{HI}, \max } \sim 47 M_{\odot} \mathrm{yr}^{-1}$ yields $E_{\text {kin }} \sim 9.4 \times 10^{42} \mathrm{erg} \mathrm{s}^{-1}$. The estimated mass of the central supermassive black hole of $3 \mathrm{C} 236$ is $\log m_{\mathrm{SMHB}} \approx 8.5 M_{\odot}$ (Mezcua et al. 2011) and implies that the maximum kinetic energy of the $\mathrm{HI}$ outflow is up to $0.02 \%$ of the Eddington luminosity. For the VLBI detected outflow, we take the integrated mass outflow rate $\left(\gtrsim 5 M_{\odot} \mathrm{yr}^{-1}\right)$, the central velocity of S3, and the full width at zero intensity (FWZI) of $500 \mathrm{~km} \mathrm{~s}^{-1}$ (Fig. 4c). This suggests that the outflow close to the nucleus has at least $4 \%$ of the maximum kinetic energy.

Recent numerical simulations of jet interaction with an inhomogeneous multiphase ISM have reached resolutions that allow a comparison with VLBI measurements (e.g., Wagner et al. 2012; Mukherjee et al. 2016, 2017; Cielo et al. 2018). This allows us to qualitatively consider the implications for our measurements. In general, the simulations demonstrated the strong effect that powerful jets have on the velocity, temperature, pressure, and density distribution of the ISM gas. When the jet hits the ISM, the gas at the shock front of the jet is accelerated to the highest velocities. While the jet continues to push through the ISM, the already accelerated gas moves outward along and transversal to the jet axis, forming a sort of expanding cocoon around the jet. This disrupts the ISM in particular in the proximity of the jet and decreases the overall density of the gas. At some point, the jet breaks through the ISM, and the gas primarily expands transversal to the jet axis. However, the expansion of the jet can also be halted if the jet power is too low and/or the density of the medium is too high. This can prevent the jet from pushing all the way through the ISM. The velocities and densities of the clouds that we measure are within the range of values expected from these simulations. Given the properties of the clumpy $\mathrm{H}_{\mathrm{I}}$ gas and the morphology of the radio emission in $3 \mathrm{C} 236$, it seems likely that we see the jet-H interaction in an already advanced stage in its evolution. However, projection effects and the undetected H I gas make it difficult to assess whether the VLBI jet has already entirely pushed through the $\mathrm{H}$ I gas.

A more quantitive comparison is difficult as most of the available simulations consider only the warm and hot ISM gas $\left(\gtrsim 10^{4} \mathrm{~K}\right)$. An exception is the recent study by Mukherjee et al. (2018) of IC 5063, which traces the cold molecular gas down to $10^{2} \mathrm{~K}$. In contrast to $3 \mathrm{C} 236$, the jet axis in IC 5063 is aligned with the disk. In this particular work, the simulations were able to reconstruct kinematic features of the cold gas as seen in observations by Morganti et al. (2015). Numerical simulations like the one performed by Mukherjee et al. (2018) are essential to understand the interaction of the radio jet and cold ISM gas.

\subsection{Disk-related Hı gas}

Struve \& Conway (2012) related the rather symmetric gradient around the deep absorption to a disk of H I gas (see Fig. 4d). They did not consider the gas detected within a distance of $<200$ mas from the nucleus to be connected to it due to the lack of spatial and kinematic structure. However, our observations reveal a velocity gradient also across the central part of the lobe (Fig. 4d). It is larger than the one at the location of the deep absorption (Fig. 4a). Because the absorption extends to the edges of the continuum and not all of the absorption is recovered, it is difficult to measure the full width of the disk. Thus, we do not calculate the properties of the disk at this point, as it would require more detailed modeling that is beyond the scope of this work.

The column density of the H I gas changes significantly across the lobe. Figure $5 \mathrm{~d}$ depicts the gas related to $\mathrm{S} 1$. Struve \& Conway (2012) reported a value of $N_{\mathrm{HI}} \approx 6.1 \times$ $10^{21} \mathrm{~cm}^{-2}$ at the location of the peak of the absorption assuming a conservative value of $T_{\text {spin }}=100 \mathrm{~K}$. This is similar to our measurements, but we find that there is variation over an order of magnitude across the lobe. The H I column densities are up to an order of magnitude lower than the column density from CO estimated by Labiano et al. (2013).

Assuming that the $\mathrm{HI}$ disk is aligned with the inner dust lane, we can estimate the height of the Hi disk using the extent of S1 in Fig. 4c. This yields $\gtrsim 200$ mas or $\gtrsim 360$ pc in projection and has to be considered a lower limit due to the undetected H I gas. Schilizzi et al. (2001) estimated an apparent inclination of the radio jet to the line of sight of $\sim 60^{\circ}$ based on the ellipticity of the host galaxy and assuming that the jets are perpendicular to the dust lanes. This yields a deprojected height of the H I disk of $z 420 \mathrm{pc}$. The major axis of the inner dust line is about $1.8 \mathrm{kpc}$ in projected size according to O'Dea et al. (2001), and the $\mathrm{CO}$ disk extends up to about $1.3 \mathrm{kpc}$. Assuming that the $\mathrm{H}$ I disk has the radial extent of the $\mathrm{CO}$ implies a thick rather 

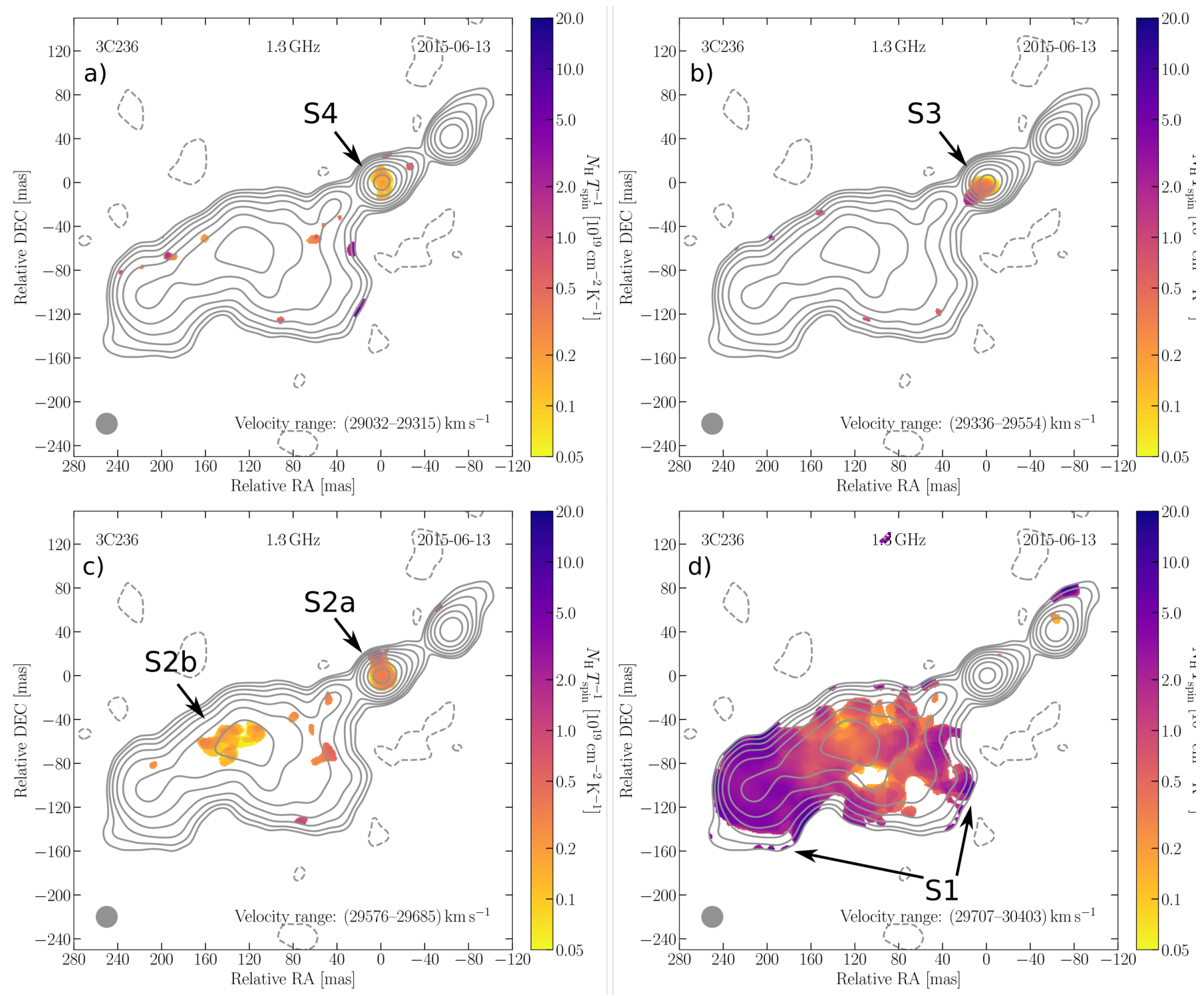

Fig. 5. Maps of $N_{\mathrm{HI}} T_{\text {spin }}^{-1}$ for the features $\mathrm{S} 1, \mathrm{~S} 2 \mathrm{a} / \mathrm{b}, \mathrm{S} 3$, and $\mathrm{S} 4$ with the same logarithmic color scale applied to all images. The velocity range is given in the bottom right corner and was chosen based on the velocity range of the features in Fig. 4. The background shows the continuum map with contours beginning at $3 \sigma_{\mathrm{VLBI}}$ and increasing logarithmically by a factor of two. The gray circle in the lower left corner depicts the synthesized beam.

than a thin disk. Nesvadba et al. (2011) also suggested an ellipsoidal configuration instead of a thin disk for the $\mathrm{H}_{2}$ gas on larger scales.

The distribution of the gas across the southeast radio lobe seen in our VLBI image and in Struve \& Conway (2012) in addition to the cospatiality with the inner dust lane provides further support for the interpretation that the morphology of the lobe is, to some extent, the result of interaction between the jet and the dust lane (O'Dea et al. 2001). Such an interaction would affect the morphology and kinematics of the H I disk. The location and kinematic properties of S2b (see Figs. $4 c$ and $d$ and Fig. 5d) could be a signature of this interaction instead of being related to outflowing gas. In this context, it is interesting that Labiano et al. (2013) required two Gaussian functions to fit the deep part of the absorption spectrum, a deep, narrow component $\left(v_{\text {hel }}=29828 \mathrm{~km} \mathrm{~s}^{-1}, F W H M \sim 80 \mathrm{~km} \mathrm{~s}^{-1}\right)$ and a shallower, broader one $\left(v_{\text {hel }}=29846 \mathrm{~km} \mathrm{~s}^{-1}, F W H M \sim 300 \mathrm{~km} \mathrm{~s}^{-1}\right)$. Further investigations are necessary and would require detailed numerical simulations of the interaction between jet and the cold ISM.

\subsection{Comparison with $4 C 12.50$}

As described in Sect. 1, the compact radio galaxy 4C 12.50 located at a redshift of 0.1217 is currently the only other powerful radio galaxy for which the H I outflow has also been studied with VLBI. In other radio galaxies, a strong $\mathrm{H}$ i outflow has also been detected and partially resolved, for instance, 3C 305 (Morganti et al. 2005a) and 3C 293 (Mahony et al. 2013). However, these observations were obtained at lower angular resolution and thus probed larger spatial scales. Therefore, we focus our comparison on 4C 12.50.

Morganti et al. (2013) showed that the H I gas is distributed on either end of its projected $200 \mathrm{pc}$ size radio structure, that is, the deep absorption is located at the northern extent of the source, while the broad outflow is cospatial with the hot spot 
Table 3. Properties of the kinematically disturbed HI clouds.

\begin{tabular}{ccccccccc}
\hline \hline Component $^{a}$ & $\begin{array}{c}N_{\mathrm{HI}} T_{\text {spin }}^{-1}{ }^{19} \\
\left(10^{19} \mathrm{~cm}^{-2} \mathrm{~K}^{-1}\right)\end{array}$ & $\begin{array}{c}N_{\mathrm{HI}}{ }^{c} \\
\left(10^{19} \mathrm{~cm}^{-2}\right)\end{array}$ & $\begin{array}{c}d^{d} \\
(\mathrm{pc})\end{array}$ & $\begin{array}{c}n_{\mathrm{HI}}{ }^{e} \\
\left(\mathrm{~cm}^{-3}\right)\end{array}$ & $\begin{array}{c}m_{\mathrm{HI}}{ }^{f} \\
\left(10^{4} M_{\odot}\right)\end{array}$ & $\begin{array}{c}v^{g} \\
\left(\mathrm{~km} \mathrm{~s}^{-1}\right)\end{array}$ & $\begin{array}{c}r_{\star}^{h} \\
(\mathrm{pc})\end{array}$ & $\begin{array}{c}\dot{M}_{\mathrm{HI}}{ }^{i} \\
\left(M_{\odot} \mathrm{yr}^{-1}\right)\end{array}$ \\
\hline S4 & 0.18 & 18 & $\lesssim 36$ & 30 & 0.65 & 640 & $\lesssim 40$ & 1.2 \\
S3 & 0.40 & 40 & $\lesssim 36$ & 60 & 1.5 & 420 & $\lesssim 40$ & 1.7 \\
S2a & 0.33 & 33 & $\lesssim 36$ & 50 & 1.2 & 150 & $\lesssim 40$ & 0.5 \\
Nucleus & 0.78 & 78 & $\lesssim 36$ & 120 & 2.8 & 640 & $\lesssim 40$ & 5 \\
S2b & 0.18 & 1.8 & $56 \times 15$ & 2 & 0.28 & 150 & 310 & 0.2 \\
\hline
\end{tabular}

Notes. ${ }^{(a)}$ Label of the kinematic component (see Fig. 4). The values for "Nucleus" were obtained after integrating over S2a, S3, and S4. ${ }^{(b)}$ H I column density normalised by spin temperature. ${ }^{(c)} \mathrm{HI}$ column density. For S4, S3, and S2a, $T_{\text {spin }}=1000 \mathrm{~K}$ is assumed, while for S2b $T_{\text {spin }}=100 \mathrm{~K}$ is assumed. ${ }^{(d)}$ Projected size of the components. For S2a, S3, and S4, a spherical geometry is assumed with an upper limit of the diameter based on the synthesized beam. For S2b, an ellipsoidal geometry is assumed, with the major and minor axis given. ${ }^{(e)}$ Density of the H I clouds. The same $T_{\text {spin }}$ values as for the column density are assumed here. ${ }^{(f)}$ Mass of the H I clouds for the chosen $T_{\text {spin }}$ values. ${ }^{(g)}$ Peak velocity of the H I clouds relative to the peak velocity of S1. ${ }^{(h)}$ Deprojected distance of the H I clouds relative to the nucleus. ${ }^{(i)}$ Mass outflow rate following Heckman (2002) for the chosen $T_{\text {spin }}$ values.

in the southern part of the source. In contrast to $3 \mathrm{C} 236$, no absorption was reported in the nuclear region and the high- and low-resolution $\mathrm{H}$ i spectrum match well, which suggests that the entire absorption has been recovered by the VLBI observation.

Morganti et al. (2013) measured a column density of the blueshifted clouds in $4 \mathrm{C} 12.50$ of $4.6 \times 10^{21} \mathrm{~cm}^{-2}$, using $T_{\text {spin }}=$ $100 \mathrm{~K}$ due to the distance of the $\mathrm{HI}$ gas to the nucleus. This is comparable to S2a, S3, and S4, even though they are located cospatial to the nucleus, that is, a higher value for $T_{\text {spin }}$ was assumed. The mass outflow rate of the $\mathrm{HI}$ in $4 \mathrm{C} 12.50$ was determined to range between $16 M_{\odot} \mathrm{yr}^{-1}$ and $29 M_{\odot} \mathrm{yr}^{-1}$. However, it is difficult to compare with $3 \mathrm{C} 236$ as we only measure lower limits. Although S2b would be better suitable for a comparison in terms of its location, its column density and total $\mathrm{H}_{\mathrm{I}}$ mass is an order of magnitude lower than what was determined for $4 \mathrm{C} 12.50$. The mass outflow rate and outflow velocity suggests a kinetic energy of about $0.02-0.03 \%$ of the Eddington luminosity for $4 \mathrm{C} 12.50$, depending also on the assumed black hole mass (Dasyra et al. 2006, 2011; Son et al. 2012). This value range is similar to what we have estimated as a possible upper limit for the kinetic energy of the outflow in 3C 236. There might also be a large difference in the total H I mass. Morganti et al. (2013) estimated a mass of $\sim 1.4 \times 10^{5} M_{\odot}$ for $4 \mathrm{C} 12.50$, which represents a lower limit as the $\mathrm{HI}$ gas could be distributed beyond the radio continuum. Struve \& Conway (2012) derived a value of 5.9-9 $\times 10^{9} M_{\odot}$, assuming the $\mathrm{H}_{\mathrm{I}}$ at the southern end of the lobe in $3 \mathrm{C} 236$ is contained within a regular rotating disk.

The differences observed between these two objects can be the result of a combination of differences in size and age of the two sources and differences in the conditions of the ISM. The gas in 3C 236 could be more settled than in $4 \mathrm{C} 12.50$. This may suggest that at the distances of the southeast radio lobe of $3 \mathrm{C} 236$ from the nuclear region of about $0.3 \mathrm{kpc}$, there are no more dense clouds and the jet has already broken through the denser gas. This would open the possibility that both sources represent different stages of evolution, at least with respect to the jet-H I interaction. 3C 236 could be further advanced in its evolution than $4 \mathrm{C} 12.50$. The ages of the two radio sources have been estimated based on cooling time to be $\sim 10^{4}$ years (4C 12.50, Morganti et al. 2013) and $\sim 10^{5}$ years (3C 236, O'Dea et al. 2001; Tremblay et al. 2010). The jet in 3C 236 could have had more time to interact with the H I dispersing the gas to greater extent. Thus, the combination of these parameters needs to be considered when the presence (or absence) of outflows and their properties are investigated.

\section{Summary and conclusion}

We have presented results on the $\mathrm{HI}$ gas distribution in the central $1 \mathrm{kpc}$ of the radio source 3C 236 as detected in absorption by milliarcsecond global VLBI and arcsecond VLA observations. We find that all of the Hi gas recovered by VLBI is contained within the nuclear region and the southeast lobe of the radio source. The VLBI data recovers a significant amount of absorption from the disk-related and the outflowing $\mathrm{H}$ i gas component compared to the lower resolution VLA data as well as about $40 \%$ of the continuum flux density. The latter implies substantial extended low-surface brightness emission that is resolved out by the high resolution of the VLBI.

For the first time, we have been able to localize part of the broad blueshifted component of the $\mathrm{H}$ I gas in 3C 236 in the form of distinct clouds located almost exclusively in the compact nuclear region, which is $\lesssim 40 \mathrm{pc}$ in size (in projection). The clouds cover a velocity range of about $600 \mathrm{~km} \mathrm{~s}^{-1}$ with respect to the peak of the disk-related $\mathrm{H}_{\text {I gas, }}$ and we estimate that they have a density of $\gtrsim 60 \mathrm{~cm}^{-3}$. There is also one cloud cospatial to the southeast lobe and well aligned with the position angle of the jet that appears to be kinematically disturbed gas. While it could be part of the H I disk, it is also possible that it resembles outflowing $\mathrm{H}$ I gas. In the latter case, its location and extended size implies a density as low as $\sim 2 \mathrm{~cm}^{-3}$. Overall, the mass outflow rate of the VLBI-detected outflowing gas is about $10 \%$ of the total mass outflow rate of $47 M_{\odot} \mathrm{yr}^{-1}$ estimated from unresolved spectra. The clouds cospatial to the nuclear region account for about $4 \%$ of the total kinetic energy of the $\mathrm{H}$ I outflow. Because 3C 236 is classified as a LERG, we consider the radio jets as the most likely driver of the outflow.

The discrepancy between the low- and high-resolution $\mathrm{HI}$ absorption spectra in combination with the distribution of the detected gas implies that the observed and undetected H I outflows are both clumpy. However, we cannot exclude the possibility of a highly diffuse gas component. A qualitative comparison with numerical simulations suggests that the interaction of the jet with the H I gas has already been going on for long time in 3C 236. In this scenario, the high-velocity gas that we do not detect has been dispersed significantly as a result of the jet interaction. Even the disk-related $\mathrm{H}$ I gas could have been affected.

We compare our results $3 \mathrm{C} 236$ to $4 \mathrm{C} 12.50$, in which no absorption was detected cospatial to the nuclear region and all of the HI was recovered by VLBI, including the jet-driven outflowing gas. The differences to 3 C 236 are very intriguing 
as it could be a sign that the gas is more settled in $3 \mathrm{C} 236$. However, the available data do not allow us to draw strong conclusions on whether the two sources represent different stages in AGN evolution. Additional data on other sources are required.

This work is part of our ongoing effort to spatially resolve the jet-driven $\mathrm{HI}$ outflow in young and restarted powerful radio galaxies. It demonstrates that great care is required when physical quantities such as density and mass of the gas are derived from unresolved spectra. This shows the need for highresolution follow-up observations of upcoming large $\mathrm{H}$ I absorption surveys conducted for example by Apertif (Oosterloo et al. 2010; Maccagni et al. 2017), MeerKat (Gupta et al. 2016), and ASKAP (Allison et al. 2015).

Acknowledgements. We thank the anonymous journal referee for their insightful comments, which improved the manuscript. RS gratefully acknowledges support from the European Research Council under the European Union's Seventh Framework Programme (FP/2007-2013)/ERC Advanced Grant RADIOLIFE-320745. EKM acknowledges support from the Australian Research Council Centre of Excellence for All-sky Astrophysics (CAASTRO), through project number CE110001020. The European VLBI Network is a joint facility of independent European, African, Asian, and North American radio astronomy institutes. Scientific results from data presented in this publication are derived from the following EVN project code: GN002. The National Radio Astronomy Observatory is a facility of the National Science Foundation operated under cooperative agreement by Associated Universities, Inc. The Long Baseline Observatory is a facility of the National Science Foundation operated under cooperative agreement by Associated Universities, Inc. The Arecibo Observatory is a facility of the National Science Foundation (NSF) operated by SRI International in alliance with the Universities Space Research Association (USRA) and UMET under a cooperative agreement. The Arecibo Observatory Planetary Radar Program is funded through the National Aeronautics and Space Administration (NASA) Near-Earth Objects Observations program. Based on observations made with the NASA/ESA Hubble Space Telescope, and obtained from the Hubble Legacy Archive, which is a collaboration between the Space Telescope Science Institute (STScI/NASA), the Space Telescope European Coordinating Facility (ST-ECF/ESA) and the Canadian Astronomy Data Centre (CADC/NRC/CSA). This research has made use of NASA's Astrophysics Data System Bibliographic Services. This research has made use of the NASA/IPAC Extragalactic Database (NED) which is operated by the Jet Propulsion Laboratory, California Institute of Technology, under contract with the National Aeronautics and Space Administration. This research made use of Astropy, a community-developed core Python package for Astronomy (Astropy Collaboration et al. 2013). This research made use of APLpy, an open-source plotting package for Python (Robitaille \& Bressert 2012).

\section{References}

Alexander, D. M., \& Hickox, R. C. 2012, New Astron. Rev., 56, 93

Allison, J. R., Sadler, E. M., Moss, V. A., et al. 2015, MNRAS, 453, 1249

Araya, E. D., Rodríguez, C., Pihlström, Y., et al. 2010, AJ, 139, 17

Associated Universities, Inc. 1999, Astrophysics Source Code Library [record ascl: 9911.003]

Astropy Collaboration (Robitaille, T. P., et al.) 2013, A\&A, 558, A33

Barthel, P. D., Miley, G. K., Jagers, W. J., Schilizzi, R. T., \& Strom, R. G. 1985 A\&A, 148, 243

Best, P. N., \& Heckman, T. M. 2012, MNRAS, 421, 1569

Böck, M. 2012, PhD Thesis, Friedrich-Alexander-Universität ErlangenNürnberg, Erlangen, Germany

Buttiglione, S., Capetti, A., Celotti, A., et al. 2010, A\&A, 509, A6

Carilli, C. L., Wrobel, J. M., \& Ulvestad, J. S. 1998, AJ, 115, 928

Cielo, S., Bieri, R., Volonteri, M., Wagner, A. Y., \& Dubois, Y. 2018, MNRAS, 477, 1336

Condon, J. J., Cotton, W. D., Greisen, E. W., et al. 1998, AJ, 115, 1693

Croton, D. J., Springel, V., White, S. D. M., et al. 2006, MNRAS, 365, 11

Dasyra, K. M., Tacconi, L. J., Davies, R. I., et al. 2006, ApJ, 638, 745

Dasyra, K. M., Ho, L. C., Netzer, H., et al. 2011, ApJ, 740, 94

Di Matteo, T., Springel, V., \& Hernquist, L. 2005, Nature, 433, 604

Fabian, A. C. 2012, ARA\&A, 50, 455

Geréb, K., Maccagni, F. M., Morganti, R., \& Oosterloo, T. A. 2015, A\&A, 575, A44
Gupta, N., Srianand, R., Baan, W., et al. 2016, Proc. MeerKAT Science: On the Pathway to the SKA (MeerKAT2016). Online at https://pos.sissa.it/ cgi-bin/reader/conf. cgi?confid=277, 14

Harrison, C. M. 2017, Nat. Astron., 1, 0165

Heckman, T. M. 2002, in Extragalactic Gas at Low Redshift, eds. J. S. Mulchaey, \& J. T. Stocke, ASP Conf. Ser., 254, 292

Heckman, T. M., \& Best, P. N. 2014, ARA\&A, 52, 589

Hill, G. J., Goodrich, R. W., \& Depoy, D. L. 1996, ApJ, 462, 163

Högbom, J. A. 1974, A\&AS, 15, 417

Holt, J., Tadhunter, C., Morganti, R., et al. 2006, MNRAS, 370, 1633

International Consortium Of Scientists 2011, Astrophysics Source Code Library [record ascl: 1107.013]

Kormendy, J., \& Ho, L. C. 2013, ARA\&A, 51, 511

Kunert-Bajraszewska, M., Gawroński, M. P., Labiano, A., \& Siemiginowska, A. 2010, MNRAS, 408, 2261

Labiano, A., García-Burillo, S., Combes, F., et al. 2013, A\&A, 549, A58

Maccagni, F. M., Morganti, R., Oosterloo, T. A., Geréb, K., \& Maddox, N. 2017, A\&A, 604, A43

Mahony, E. K., Morganti, R., Emonts, B. H. C., Oosterloo, T. A., \& Tadhunter, C. 2013, MNRAS, 435, L58

McNamara, B. R., \& Nulsen, P. E. J. 2007, ARA\&A, 45, 117

Meyer, M., Robotham, A., Obreschkow, D., et al. 2017, PASA, 34, e052

Mezcua, M., Lobanov, A. P., Chavushyan, V. H., \& León-Tavares, J. 2011, A\&A, 527, A38

Morganti, R. 2017, Front. Astron. Space Sci., 4, 42

Morganti, R., Oosterloo, T., \& Tsvetanov, Z. 1998, AJ, 115, 915

Morganti, R., Oosterloo, T. A., Tadhunter, C. N., van Moorsel, G., \& Emonts, B. 2005a, A\&A, 439, 521

Morganti, R., Tadhunter, C. N., \& Oosterloo, T. A. 2005b, A\&A, 444, L9

Morganti, R., Fogasy, J., Paragi, Z., Oosterloo, T., \& Orienti, M. 2013, Science, 341,1082

Morganti, R., Oosterloo, T., Oonk, J. B. R., Frieswijk, W., \& Tadhunter, C. 2015, A\&A, 580, A1

Morganti, R., Veilleux, S., Oosterloo, T., Teng, S. H., \& Rupke, D. 2016, A\&A, 593, A30

Mukherjee, D., Bicknell, G. V., Sutherland, R., \& Wagner, A. 2016, MNRAS, 461, 967

Mukherjee, D., Bicknell, G. V., Sutherland, R., \& Wagner, A. 2017, MNRAS, 471, 2790

Mukherjee, D., Wagner, A. Y., Bicknell, G. V., et al. 2018, MNRAS, 476, 80

Nesvadba, N. P. H., Boulanger, F., Lehnert, M. D., Guillard, P., \& Salome, P. 2011, A\&A, 536, L5

Nyland, K., Young, L. M., Wrobel, J. M., et al. 2016, MNRAS, 458, 2221

O'Dea, C. P. 1998, PASP, 110, 493

O'Dea, C. P., Koekemoer, A. M., Baum, S. A., et al. 2001, AJ, 121, 1915

Oosterloo, T. A., Morganti, R., Tzioumis, A., et al. 2000, AJ, 119, 2085

Oosterloo, T., Verheijen, M., \& van Cappellen, W. 2010, in ISKAF 2010 Science Meeting, 43

Orienti, M. 2016, Astron. Nachr., 337, 9

Peck, A. B., \& Taylor, G. B. 2001, ApJ, 554, L147

Peck, A. B., Taylor, G. B., \& Conway, J. E. 1999, ApJ, 521, 103

Perley, R. A., \& Butler, B. J. 2013, ApJS, 204, 19

Robitaille, T., \& Bressert, E. 2012, Astrophysics Source Code Library [record ascl: 1208.017]

Sault, R. J., Teuben, P. J., \& Wright, M. C. H. 1995, in Astronomical Data Analysis Software and Systems IV, eds. R. A. Shaw, H. E. Payne, \& J. J. E. Hayes, ASP Conf. Ser., 77, 433

Sault, R. J., Teuben, P., \& Wright, M. C. H. 2011, Astrophysics Source Code Library [record ascl: 1106.007]

Schilizzi, R. T., Tian, W. W., Conway, J. E., et al. 2001, A\&A, 368, 398

Shepherd, M. 2011, Astrophysics Source Code Library [record ascl: 1103.001]

Shepherd, M. C., Pearson, T. J., \& Taylor, G. B. 1994, BAAS, 26, 987

Silk, J., \& Rees, M. J. 1998, A\&A, 331, L1

Son, D., Woo, J.-H., Kim, S. C., et al. 2012, ApJ, 757, 140

Struve, C., \& Conway, J. E. 2010, A\&A, 513, A10

Struve, C., \& Conway, J. E. 2012, A\&A, 546, A22

Tadhunter, C. 2016, A\&ARv, 24, 10

Taylor, G. B., Hough, D. H., \& Venturi, T. 2001, ApJ, 559, 703

Tremblay, G. R., O'Dea, C. P., Baum, S. A., et al. 2010, ApJ, 715, 172

van Gorkom, J. H., Knapp, G. R., Ekers, R. D., et al. 1989, AJ, 97, 708

Veilleux, S., Cecil, G., \& Bland-Hawthorn, J. 2005, ARA\&A, 43, 769

Vermeulen, R. C., Ros, E., Kellermann, K. I., et al. 2003, A\&A, 401, 113

Vermeulen, R. C., Labiano, A., Barthel, P. D., et al. 2006, A\&A, 447, 489

Wagner, A. Y., Bicknell, G. V., \& Umemura, M. 2012, ApJ, 757, 136

Wagner, A. Y., Bicknell, G. V., Umemura, M., Sutherland, R. S., \& Silk, J. 2016, Astron. Nachr., 337, 167

Willis, A. G., Strom, R. G., \& Wilson, A. S. 1974, Nature, 250, 625 